\title{
Performance evaluation of the simplified spherical harmonics approximation for cone-beam X-ray luminescence computed tomography imaging
}

\author{
Haibo Zhang*, Guohua Geng**, Yanrong Chen*, Fengjun Zhao*, \\ Yuqing Hou*, Huangjian Yi*, Shunli Zhang*, \\ Jingjing $\mathrm{Yu}^{\dagger}$ and Xiaowei $\mathrm{He}^{*, \S}$ \\ ${ }^{*}$ School of Information Sciences and Technology \\ Northwest University, Xi'an \\ Shannxi 710027, P. R. China \\ ${ }^{\dagger}$ School of Physics and Information Technology \\ Shaanxi Normal University \\ Xi'an, Shannxi 710062, P. R. China \\ †ghgeng@nwu.edu.cn \\ \$hexw@nwu.edu.cn
}

Received 23 September 2016

Accepted 14 December 2016

Published 25 January 2017

\begin{abstract}
As an emerging molecular imaging modality, cone-beam X-ray luminescence computed tomography (CB-XLCT) uses X-ray-excitable probes to produce near-infrared (NIR) luminescence and then reconstructs three-dimensional (3D) distribution of the probes from surface measurements. A proper photon-transportation model is critical to accuracy of XLCT. Here, we presented a systematic comparison between the common-used Monte Carlo model and simplified spherical harmonics $\left(S P_{\mathrm{N}}\right)$. The performance of the two methods was evaluated over several main spectrums using a known XLCT material. We designed both a global measurement based on the cosine similarity and a locally-averaged relative error, to quantitatively assess these methods. The results show that the $S P_{3}$ could reach a good balance between the modeling accuracy and computational efficiency for all of the tested emission spectrums. Besides, the $S P_{1}$ (which is equivalent to the diffusion equation (DE)) can be a reasonable alternative model for emission wavelength over $692 \mathrm{~nm}$. In vivo experiment further demonstrates the reconstruction performance of the $\mathrm{SP}_{3}$ and $\mathrm{DE}$. This study would provide a valuable guidance for modeling the photon-transportation in CB-XLCT.
\end{abstract}

Keywords: Cone-beam X-ray luminescence computed tomography; photon-transportation model; simplified spherical harmonics approximation; diffusion equations.

$\S_{\text {Corresponding author. }}$

This is an Open Access article published by World Scientific Publishing Company. It is distributed under the terms of the Creative Commons Attribution 4.0 (CC-BY) License. Further distribution of this work is permitted, provided the original work is properly cited. 


\section{Introduction}

X-ray luminescence computed tomography (XLCT) is an attractive imaging modality, with the potential to improve the characterization and treatment response of small tumors. ${ }^{1,2}$ The deep embedded nanophosphor materials in biological tissues emit near-infrared (NIR) luminescence light signals when stimulated by X-rays. ${ }^{3}$ These materials, such as $\mathrm{NaGdF}_{4}: \mathrm{Eu}, \quad \mathrm{GOS}: \mathrm{Eu}, \mathrm{Gd}_{2} \mathrm{O}_{2} \mathrm{~S}: \mathrm{Eu}, \mathrm{EU}_{2} \mathrm{O}_{3}$, and $\mathrm{Gd}_{2} \mathrm{O}_{2} \mathrm{~S}: \mathrm{Tb}$, have been successfully applied to past XLCT study. Generally, these materials contain about 12 emission peaks $(607,615,618,620,623$, 627, 667, 692, 700, 703, 720 and $802 \mathrm{~nm}) .{ }^{4-9}$

Compared with conventional pre-clinical molecular imaging techniques, i.e., fluorescence molecular tomography $(\mathrm{FMT})^{10,11}$ and bioluminescence tomography (BLT), ${ }^{12,13}$ the advantages of XLCT are manifested in the avoidance of a significant background noise and the ability of imaging deeper tissue. ${ }^{3-6,9}$ XLCT imaging could be achieved either by the pencil-beam XLCT (PB-XLCT) ${ }^{5}$ or the conebeam XLCT (CB-XLCT) methodologies. ${ }^{6}$ The PBXLCT has high-resolution at depth but with long time of data acquisition (typically, $\geq 624 \mathrm{~s}$ ) $.{ }^{9} \mathrm{Com}-$ pared with PB-XLCT, the CB-XLCT can decrease the scanning time and fully utilize the X-ray dose by using a cone beam X-ray source (multi-view data scanning: $134 \mathrm{~s}$ or single view data scanning: $\leq 1 \mathrm{~s}) .5,9$

The establishment of an accurate and practical photon-transportation model is key in XLCT. In optical imaging, photon propagation in tissues can be depicted by the Monte Carlo (MC) method, or by the radiative transfer equation (RTE) theory. ${ }^{14-16}$ However, both methods are computationally expensive. The diffusion equations (DE), a loworder approximation of RTE, have been extensively used for three-dimensional (3D) optical imaging (e.g., BLT, FMT, and XLCT. ${ }^{4-6,10-13}$ ) because of its computational efficiency. However, DE is not applicable in tissues with low-scattering-low-absorption and low-scattering-high-absorption. ${ }^{17}$ Besides, it is not suitable to describe the photon transportation in regions near the light source ${ }^{14,15}$ by DE.

To get a more accurate approximation of RTE, a high-order simplified spherical harmonics $\left(S P_{\mathrm{N}}\right)$ approximation has been developed. It exhibits better performance than the DE, thus was introduced in optical imaging. ${ }^{18}$ Some studies have proved that the $S P_{\mathrm{N}}$ models can efficiently improve the imaging accuracy, e.g., BLT, FMT. ${ }^{19-21}$ Further, some hybrid models based on the $S P_{\mathrm{N}}$ have also been proposed to exhibit the applicability in optical imaging. ${ }^{22,23}$ However, $S P_{\mathrm{N}}$ models do not converge to the exact RTE approximation when $N \rightarrow \infty .^{18}$ Besides, $S P_{\mathrm{N}}$ models yield different best solutions when using different $N$ and different XLCT probes. These two facts lead to the need of individual determination of the optimal $N$. Therefore, it is necessary to evaluate the performance of $S P_{\mathrm{N}}$ with different $N$ values for CB-XLCT imaging.

In this paper, we investigated the performance and applicability of the $S P_{\mathrm{N}}$ models for CB-XLCT imaging in detail. More specifically, the performance of the $S P_{\mathrm{N}}$ models was compared with the MC method under the finite element method (FEM), from order $N=1$ up to order $N=7$ over several main spectrums for the known XLCT probes. Then, an in vivo data was used to further evaluate reconstruction performance of the $S P_{3}$ and $S P_{1}$.

The outline of this paper is as follows. Section 2 presents the theory and method of this paper. Then, the experimental results were demonstrated based on a $3 \mathrm{D}$ digital mouse model and an in vivo experiment in Sec. 3. Finally, we conclude the paper and discuss relevant issues in Sec. 4.

\section{Method}

\section{1. $\quad S P_{\mathrm{N}}$ model}

Higher-order $S P_{\mathrm{N}}$ models result in a significant increase in time-consumption. Thus, the $S P_{\mathrm{N}}$ up to $S P_{7}$ were investigated. Equations (1) give the $3 \mathrm{D}$ $S P_{7}$ equations. The $S P_{5}, S P_{3}$ and $S P_{1}$ (equals to $\mathrm{DE})$ can be easily obtained from $S P_{7} \cdot{ }^{18}$

$$
\left\{\begin{array}{l}
-\nabla \cdot \frac{1}{3 \mu_{a 1}} \nabla \varphi_{1}+\mu_{a} \varphi_{1}=S+\left(\frac{2}{3} \mu_{a}\right) \varphi_{2}-\left(\frac{8}{15} \mu_{a}\right) \varphi_{3} \\
+\left(\frac{16}{35} \mu_{a}\right) \varphi_{4}-\nabla \cdot \frac{1}{7 \mu_{a 3}} \nabla \varphi_{2}+\left(\frac{4}{9} \mu_{a}+\frac{5}{9} \mu_{a 2}\right) \varphi_{2} \\
=-\frac{2}{3} S+\left(\frac{2}{3} \mu_{a}\right) \varphi_{1}+\left(\frac{16}{45} \mu_{a}+\frac{4}{9} \mu_{a 2}\right) \varphi_{3} \\
-\left(\frac{32}{105} \mu_{a}+\frac{8}{21} \mu_{a 2}\right) \varphi_{4}-\nabla \cdot \frac{1}{11 \mu_{a 5}} \nabla \varphi_{3} \\
+\left(\frac{64}{225} \mu_{a}+\frac{16}{45} \mu_{a 2}+\frac{9}{25} \mu_{a 4}\right) \mu_{a 3} \\
=\frac{8}{15} S-\left(\frac{8}{15} \mu_{a}\right) \varphi_{1}+\left(\frac{16}{45} \mu_{a}+\frac{4}{9} \mu_{a 2}\right) \varphi_{2} \\
+\left(\frac{128}{525} \mu_{a}+\frac{32}{105} \mu_{a 2}+\frac{54}{175} \mu_{a 4}\right) \varphi_{4}-\nabla \cdot \frac{1}{15 \mu_{a 7}} \nabla \varphi_{3} \\
+\left(\frac{256}{1225} \mu_{a}+\frac{64}{245} \mu_{a 2}+\frac{324}{1225} \mu_{a 4}+\frac{54}{175} \mu_{a 6}\right) \varphi_{4} \\
=-\frac{16}{35} S+\left(\frac{16}{35} \mu_{a}\right) \varphi_{1}-\left(\frac{32}{105} \mu_{a}+\frac{8}{21} \mu_{a 2}\right) \varphi_{2} \\
+\left(\frac{128}{525} \mu_{a}+\frac{32}{105} \mu_{a 2}+\frac{54}{175} \mu_{a 4}\right) \varphi_{3}
\end{array}\right.
$$


where $\mu_{a n}=\mu_{t}(x)-\mu_{s}(x) g^{n}$ is the $n$th order absorption coefficient, $\mu_{s}$ is the scattering coefficient, and $g$ is the anisotropy parameter. $S$ is the XLCT source density, and $\varphi_{n}$ is the composites of the $n$th order Legendre moments, which is defined as follows:

$$
\left\{\begin{array}{l}
\varphi_{1}=\phi_{0}+2 \phi_{2}, \\
\varphi_{2}=3 \phi_{2}+4 \phi_{4}, \\
\varphi_{3}=5 \phi_{4}+6 \phi_{6}, \\
\varphi_{4}=7 \phi_{6} .
\end{array}\right.
$$

By setting $\phi_{6}=0$ and solving Eq. (1), the $S P_{5}$ can be obtained. Similarly, the $S P_{3}$ can be obtained by setting $\phi_{6}=\phi_{4}=0$ and $\operatorname{DE}\left(S P_{1}\right)$ can be obtained by setting $\phi_{6}=\phi_{4}=\phi_{2}=0$. The boundary conditions of the $S P_{\mathrm{N}}$ model can be found in Klose' work. ${ }^{18}$

For the case of $N=1$ and $N=3$, the $\mathrm{DE}\left(S P_{1}\right)$ and $S P_{3}$ were shown in Eqs. (3) and (4) respectively:

$$
-\nabla \cdot \frac{1}{3 \mu_{a 1}} \nabla \phi_{0}(r)+\mu_{a}(r) \phi_{0}(r)=S(r)
$$

where $r$ is the position vector.

$$
\left\{\begin{array}{l}
-\nabla \cdot \frac{1}{3 \mu_{a 1}} \nabla \phi_{1}(r)+\mu_{a} \phi_{1}(r)-\left(\frac{2}{3} \mu_{a}\right) \phi_{2}(r)=S, \\
-\nabla \cdot \frac{1}{7 \mu_{a 3}} \nabla \phi_{2}(r)+\left(\frac{4}{9} \mu_{a}+\frac{5}{9} \mu_{a 2}\right) \phi_{2}(r) \\
-\frac{2}{3} \mu_{a} \phi_{1}(r)=-\frac{2}{3} S .
\end{array}\right.
$$

Using the FEM and Green's first formula,${ }^{24}$ the $S P_{\mathrm{N}}$ model can be discretized to the matrix-form equation. For the case of $N=3, S P_{3}$ matrix-form equation can be obtained:

$$
\left[\begin{array}{ll}
M_{1 \varphi_{1}} & M_{1 \varphi_{2}} \\
M_{2 \varphi_{1}} & M_{2 \varphi_{2}}
\end{array}\right]\left[\begin{array}{l}
\varphi_{1} \\
\varphi_{2}
\end{array}\right]=\left[\begin{array}{c}
S \\
-\frac{2}{3} S
\end{array}\right] .
$$

In Eq. (5), the unknown source $S$ is approximated as $S(r) \approx \sum_{p=1}^{T} \Phi_{p} X_{p} \nu_{p}(r)$, where $X_{p}$ is the unknown material distribution, $\nu_{p}(r)$ is the base function for node $p$ and $T$ is the total number of the discretized nodes. Then, the following equation can be obtained:

$$
\left[\begin{array}{ll}
M_{1 \varphi_{1}} & M_{1 \varphi_{2}} \\
M_{2 \varphi_{1}} & M_{2 \varphi_{2}}
\end{array}\right]\left[\begin{array}{l}
\varphi_{1} \\
\varphi_{2}
\end{array}\right]=\left[\begin{array}{ll}
E_{\Phi} & 0 \\
0 & E_{\Phi}
\end{array}\right]\left[\begin{array}{c}
X \\
-\frac{2}{3} X
\end{array}\right]
$$

\section{2. $C B-X L C T$ imaging model based on the $S P_{\mathrm{N}}$ method}

The CB-XLCT imaging model includes three steps which are shown as follows:

First, X-rays are emitted from the cone-beam X-ray source. According to Beer-Lambert's law, $\mathrm{X}$-rays suffer from attenuation in tissues as follows:

$$
X(r)=X_{0} \exp \left\{-\int_{r_{0}}^{r} \mu_{x}(\tau) d \tau\right\},
$$

where $X(r)$ is the $\mathrm{X}$-ray source intensity at position $r, X_{0}$ is the $\mathrm{X}$-ray source intensity at the initial position $r_{0}$, and $\mu_{x}$ is the X-ray attenuation coefficient which can be obtained by the attenuationbased CT technique.

Second, XLCT probes excited by X-rays emit visible or NIR light. This can be expressed as follows:

$$
S(r)=\varepsilon X(r) \rho(r),
$$

where $\rho$ is the material density, and $\varepsilon$ is the light yield.

Finally, the CCD camera captures the emitted photons which reach the surface of biological tissues. The photon-transportation in biological tissues can be modeled by the $S P_{\mathrm{N}}$ method and expressed as follows:

$$
J^{+}=A_{S P_{N}} X,
$$

where $J^{+}$is the exiting partial current measurement.

For the reconstruction, due to the ill-posedness of CB-XLCT problem and the noise in the measured data, it is impractical to solve the unknown material distribution $X$ directly. ${ }^{6,9}$ Meanwhile, XLCT materials are sparsely distributed. Therefore, the $L_{1}$ regularization can be applied to CBXLCT imaging as follows:

$$
\min \left\|A_{S P_{N}} X-J^{+}\right\|_{2}^{2}+\tau\|X\|_{1},
$$

where $\tau$ is the regularization parameter.

\section{Experiments and Results}

\subsection{Forward simulation}

To test the accuracy of the $S P_{\mathrm{N}}$ model based on 12 emission spectrums of the known XLCT materials for CB-XLCT imaging, we conducted numerical experiments of forward modeling. These experiments compare the $S P_{\mathrm{N}}$ model including $\mathrm{DE}$ 


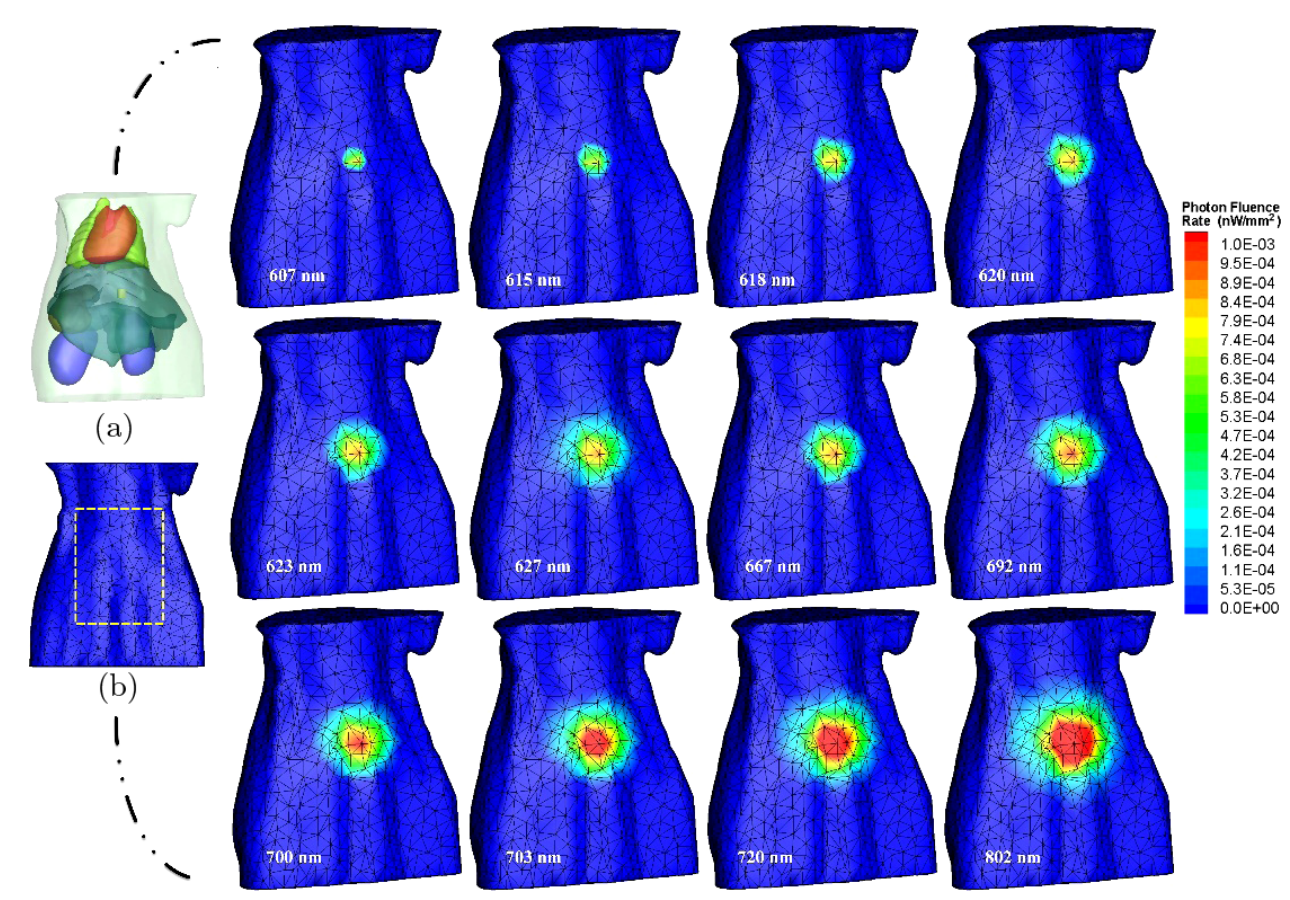

(c)

Fig. 1. The results of the MC method: (a) The tested mouse model used for comparison, containing six organs: heart, lung, stomach, liver, kidney and muscle. (b) A local area of the surface where the photo distributes densely. (c) The simulation results of 12 spectrums by the MC method based on the MOSE platform. ${ }^{15}$

$\left(S P_{1}\right), S P_{3}, S P_{5}, S P_{7}$ with the MC method which is known as the gold standard in photon-transportation modeling. ${ }^{16,25}$ All experiments were performed on a desktop computer with $3.40 \mathrm{GHz}$ Intel $\mathbb{R}$ Xeon $($ P Processor E3-1231 and 12 GB RAM.

In the numerical experiments, a digital mouse model $^{26}$ was discretized into tetrahedral mesh (10151 nodes and 55581 tetrahedrons). A cylindrical light source with a radius of $0.5 \mathrm{~mm}$ and a height of $1.5 \mathrm{~mm}$ was positioned at $(17.8,6.6,16.4) \mathrm{mm}$ to simulate different materials, as shown in Fig. 1(a). A local area of the surface where the photo distribute densely was shown in Fig. 1(b). The optical properties $\mu_{a}$ and $\mu_{s}$ of different organs were calculated by Alexandrakis' work. ${ }^{27}$ The X-ray source was set to $50 \mathrm{kVp}$ voltage and $1 \mathrm{~mA}$ current. The mass of the simulated target was set to $1 \mu \mathrm{g}$. By setting the photon number as $10^{8}$, the simulation results of MC method from the software of molecular optical simulation environment $(\mathrm{MOSE})^{16}$ are shown in Fig. 1(c).

Figure 2 demonstrates the results of three examples each with a commonly used spectrum in CB-XLCT imaging (i.e., 627, 700 and $802 \mathrm{~nm}$, respectively). Particularly, we computed the normalized surface energy of two extracted lines $(x=17 \mathrm{~mm}, z=16 \mathrm{~mm})$, and sorted the surface energy of five models in descending order. Figure 3 is the corresponding results of the top 50 largest points of the five models based on two lines. In comparison with MC method, it is clear that $S P_{3}$, $S P_{5}$ and $S P_{7}$ models get better results than DE $\left(S P_{1}\right)$.

To quantitatively assess the simulation results, a global measurement based on the cosine similarity $G_{\cos }$ was designed. It is noted that the greater $G_{\mathrm{cos}}$ is, the greater the similarity of the two models is.

$$
G_{\mathrm{COS}}=\frac{\sum_{k=1}^{M} j_{1, k} \bullet j_{2, k}^{\mathrm{MC}}}{\sqrt{\sum_{k=1}^{M}\left(j_{1, k}\right)^{2}} \cdot \sqrt{\sum_{k=1}^{M}\left(j_{1, K}^{\mathrm{MC}}\right)^{2}}},
$$

where $j_{1, k}$ is flux of the $k$ th nodes, $j_{2, k}^{\mathrm{MC}}$ is the corresponding MC's flux and $M$ is the total number of nodes on the surface.

Meanwhile, a locally-averaged relative error $\left(L_{\text {are }}\right)$ is designed to compare the density distribution of surface flux between $\mathrm{MC}$ and other forward models. The local area is shown in Fig. $1(\mathrm{~b}) . L_{\text {are }}$ is 


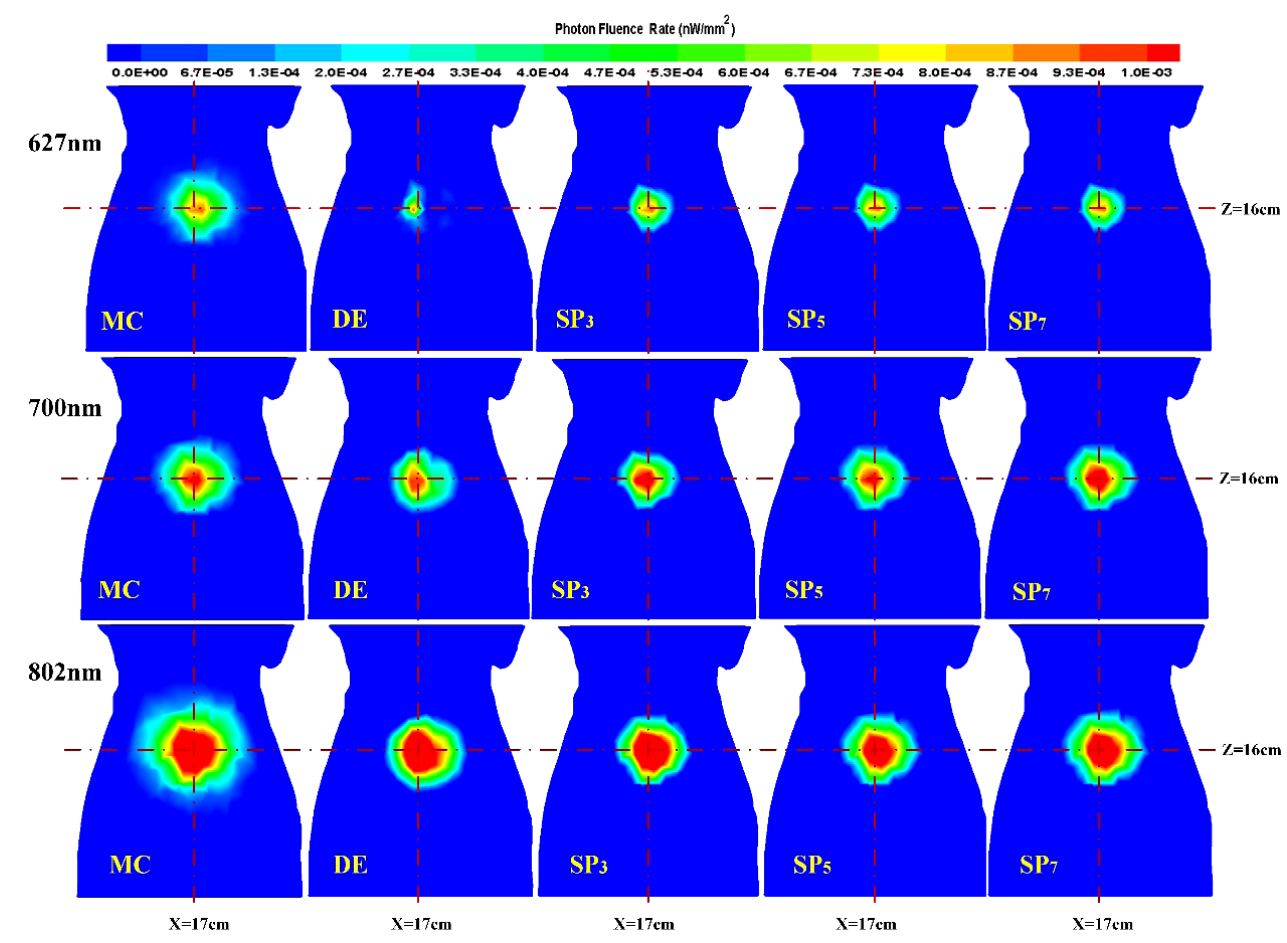

Fig. 2. Three examples of the results for 627,700 and $802 \mathrm{~nm}$ which used different forward models.

defined as follows:

$$
L_{\text {are }}=\frac{\sum_{i=1}^{N_{L}} \operatorname{abs}\left(j_{i}^{\mathrm{MC}}-j_{i}\right) / \max \left(j_{i}^{\mathrm{MC}}\right)}{N_{L}},
$$

where $j_{i}^{\mathrm{MC}}$ and $j_{i}$ are flux of the ith nodes on the surface of the MC and other forward models respectively, and $N_{L}$ is the total number of nodes on the local area.

The comparative results of $G_{\cos }$ and $L_{\text {are }}$ for different models are shown in Fig. 4. Tables 1 and 2 give numerical results of $G_{\mathrm{cos}}$ and $L_{\text {are }}$, respectively. Table 3 gives the average time-consumption of five models.

As is illustrated in Fig. 4(a) and Table 1, the $G_{\text {cos }}$ of all models were below $70 \%$ when the spectrum was under $627 \mathrm{~nm}$. Meanwhile, a great improvement of $G_{\text {cos }}$ can be obtained by the increase of wavelength. The $S P_{3}, S P_{5}$ and $S P_{7}$ models were superior than DE $\left(S P_{1}\right)$ over all 12 spectrums, and their $G_{\mathrm{cos}}$ values were almost the same. Due to high computational cost of $S P_{5}$ and $S P_{7}$ models as listed in Table 3, the two models were discarded in the following comparisons and analysis. The difference of $G_{\text {cos }}$ between DE $\left(S P_{1}\right)$ and $S P_{3}$ model varied from $0.11 \%$ to $17.58 \%$ when spectrum was between $607 \mathrm{~nm}$ and $802 \mathrm{~nm}$, and dropped to $0.94 \%$ when spectrum came to $692 \mathrm{~nm}$. This was mainly caused by the high-scattering-low-absorption properties of soft tissue. For the results of $L_{\text {are }}$, we can see in Fig. 4(b) and Table 2 that tendency of the four $S P_{\mathrm{N}}$ models are similar from $607 \mathrm{~nm}$ to $802 \mathrm{~nm}$, and the error of $\mathrm{DE}\left(S P_{1}\right)$ is larger than that of the other three models. Compared with DE $\left(S P_{1}\right)$ model, the $S P_{3}$ model was more superior than over all spectrums, and their difference of $L_{\text {are }}$ dropped to $0.33 \%$ when spectrum increased to $692 \mathrm{~nm}$.

\subsection{In vivo experiments}

In this section, we evaluated the performance of $\mathrm{DE}$ $\left(S P_{1}\right)$ and $S P_{3}$ in inverse reconstruction with in vivo experimental data. ${ }^{6}$ In the experiments, a female eight-week-old mouse was used. The homogeneous absorption coefficient was $0.3 \mathrm{~mm}^{-1}$ and the reduced scattering coefficient was $10 \mathrm{~mm}^{-1} .{ }^{28} \mathrm{~A}$ plastic capillary (radius: $1 \mathrm{~mm}$, height: of $2 \mathrm{~mm}$ ) with $\mathrm{Gd}_{2} \mathrm{O}_{2} \mathrm{~S}: \mathrm{Tb}$ nanophosphors filled in was implanted into the abdomen of the mouse. The light yield $\varepsilon$ of $\mathrm{Gd}_{2} \mathrm{O}_{2} \mathrm{~S}$ : $\mathrm{Tb}$ nanophosphors was $5 \times 10^{9}$ photons/Gy $\times$ mg. The concentration of $\mathrm{Gd}_{2} \mathrm{O}_{2} \mathrm{~S}: \mathrm{Tb}$ nanophosphors was $0.015 \mathrm{~g} / \mathrm{mL}$. The voltage and current of X-ray source (Apogee, Oxford 


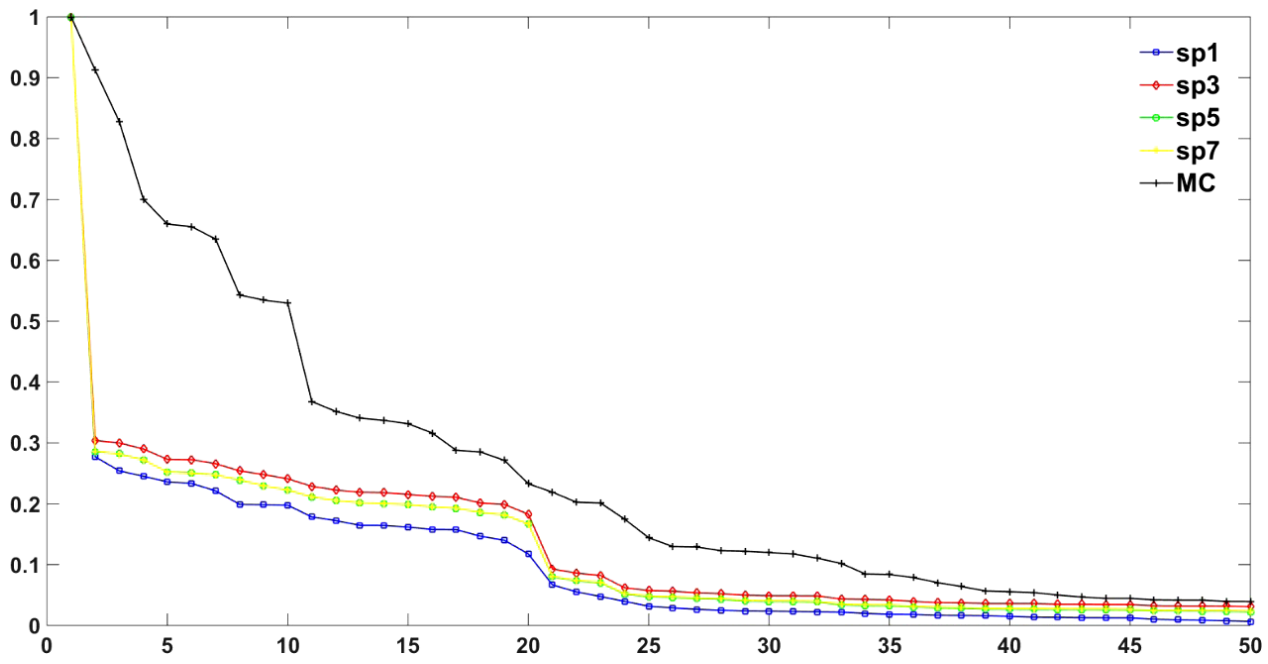

(a)

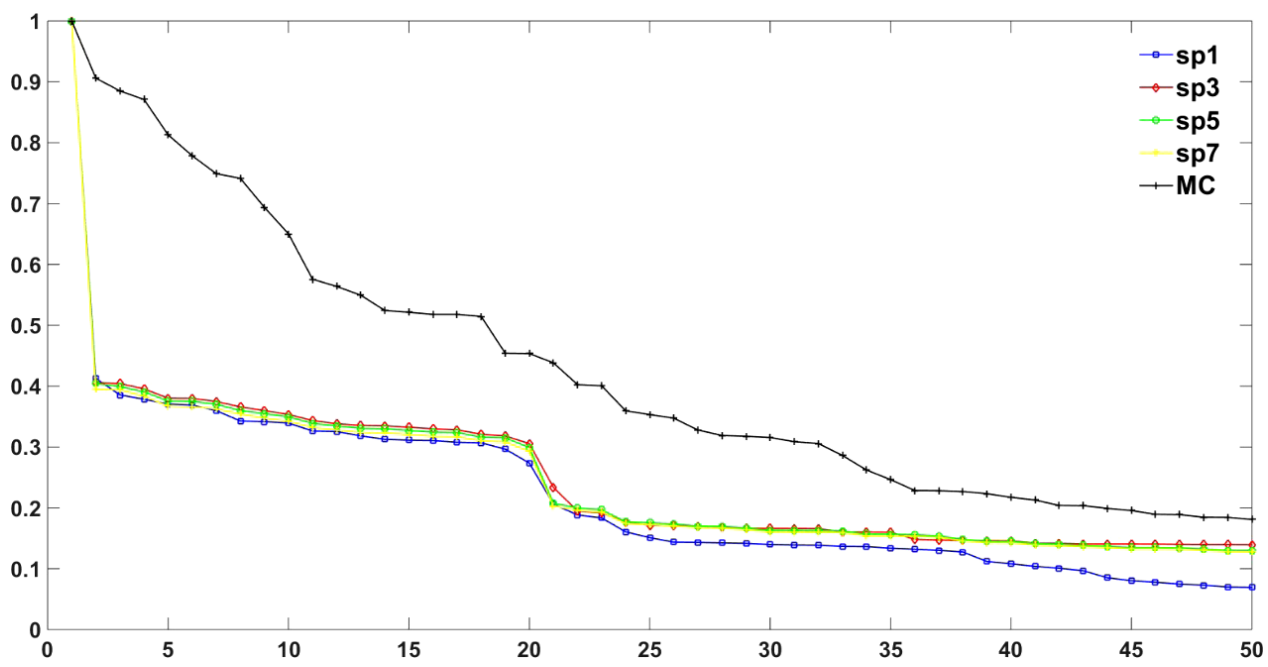

(b)

Fig. 3. The corresponding results of the largest 50 points of the five models based on the two lines. (a) The result of $x=17 \mathrm{~cm}$. (b) The result of $z=16 \mathrm{~cm}$.

Instruments, USA) with a micro $55 \mathrm{~mm} \mathrm{f} / 2.8$ lens (Nikkor, Nikon, Japan) were set to $50 \mathrm{kVp}$ and $1 \mathrm{~mA}$, respectively. A highly sensitive CCD camera (PIXIS 2048, Princeton Instruments, USA) with a micro $55 \mathrm{~mm}$ f/2.8 lens (Nikkor, Nikon, Japan) was mounted vertical to the X-ray axis to capture the optical data $(620 \mathrm{~nm})$. The CCD binning was set to $2 \times 2$ and the integrating time of CCD was set to $30 \mathrm{~s}$. The micro-CT imaging was performed (360 views with $1^{\circ}$ intervals) using a flat-panel detector (C7921CA-02, Hamamatsu, Japan) to get the structural information. The $3 \mathrm{D}$ reconstruction of $\mathrm{XCT}$ projections was performed employing the Feldkamp-Davis-Kress (FDK) method. ${ }^{29}$
The mouse was excited by a cone beam X-ray source from different directions with $45^{\circ}$ intervals to acquire the luminescent photons. Figure 5(a) shows the distribution of forward photon on the surface. The micro-CT result at $z=6.8 \mathrm{~mm}$ is demonstrated in Fig. 5(b).

In reconstruction, we used the incomplete variables truncated conjugate gradient (IVTCG) algorithm proposed in our work. ${ }^{12}$ The IVTCG algorithm is based on $L_{1}$ regularization framework, and has been widely applied to optical molecular imaging, e.g., BLT, FMT and XLCT. ${ }^{6,30,31}$ We used location error (LE), recovered density and relative quantity error to analyze the results. ${ }^{6}$ 


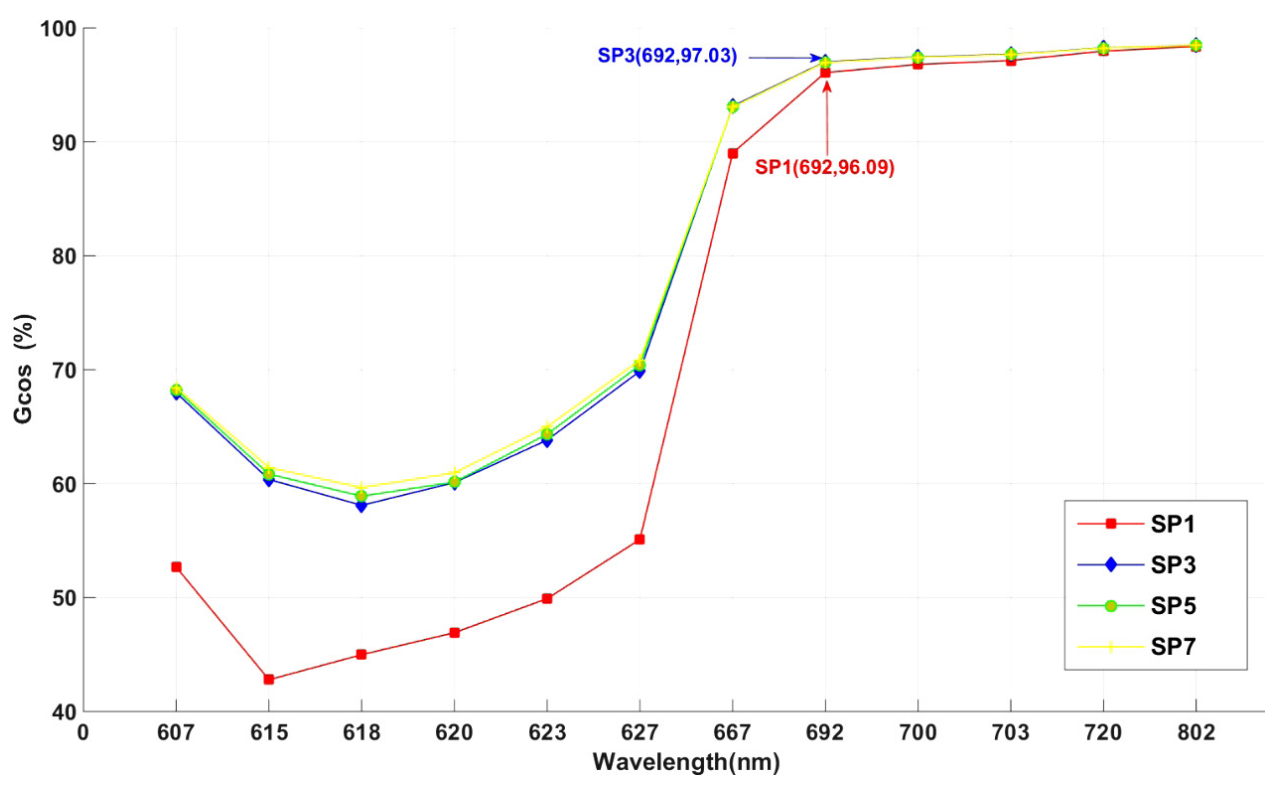

(a)

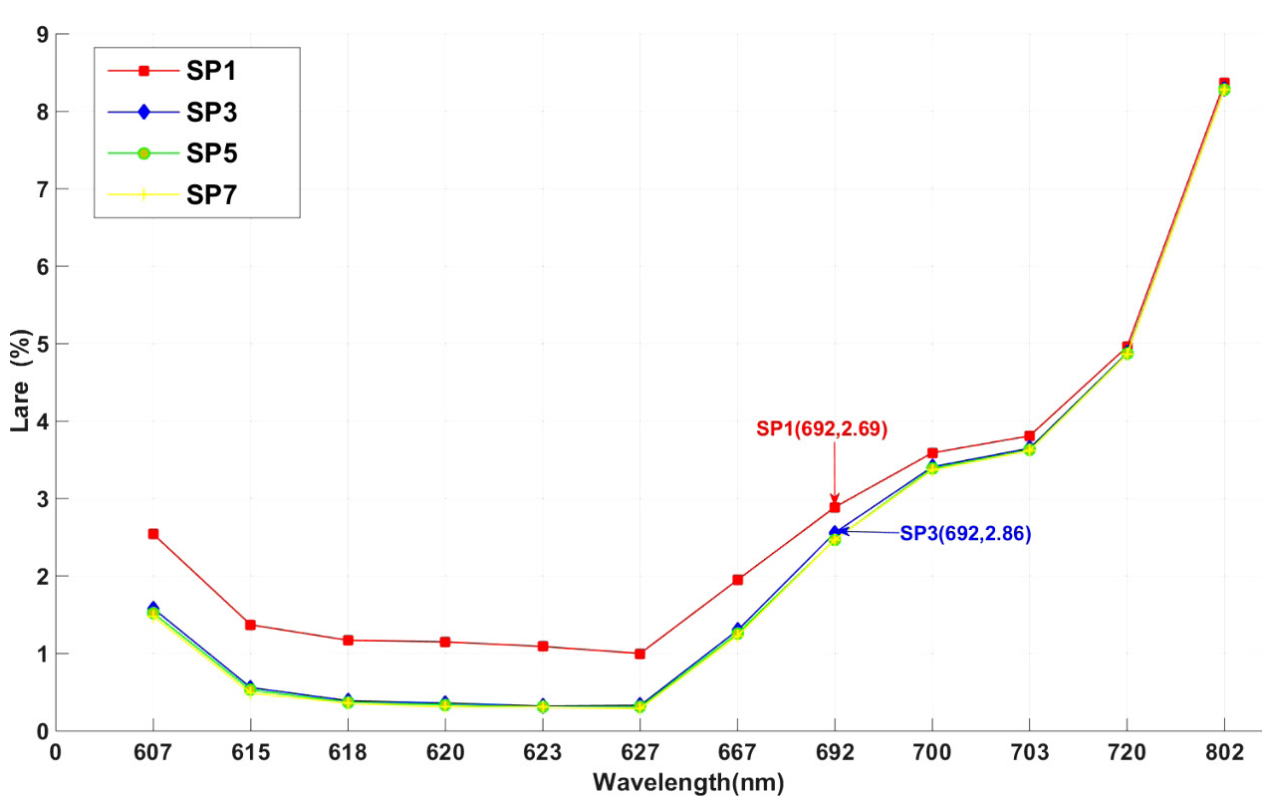

(b)

Fig. 4. The results of $G_{\mathrm{cos}}$ and $L_{\text {are }}$ : (a) The global comparison results between $S P_{\mathrm{N}}$ model and MC method based on cosine similarity. (b) The local average relative error between $S P_{\mathrm{N}}$ model and $\mathrm{MC}$ method.

Table 1. The $G_{\mathrm{cos}}$ between the $S P_{\mathrm{N}}$ model results and MC model results.

\begin{tabular}{lcccccccccccc}
\hline$G_{\text {cos }}(\%)$ & 607 & 615 & 618 & 620 & 623 & 627 & 667 & 692 & 700 & 703 & 720 & 802 \\
\hline $\mathrm{DE}\left(S P_{1}\right)$ & 52.67 & 42.79 & 44.99 & 46.92 & 49.91 & 55.10 & 89.02 & 96.09 & 96.80 & 97.41 & 97.79 & 98.38 \\
$S P_{3}$ & 67.91 & 60.37 & 58.10 & 60.10 & 63.83 & 69.87 & 93.15 & 97.03 & 97.46 & 97.69 & 98.26 & 98.49 \\
$S P_{5}$ & 68.24 & 60.84 & 58.90 & 60.14 & 64.34 & 70.39 & 93.05 & 96.98 & 97.42 & 97.66 & 98.24 & 98.48 \\
$S P_{7}$ & 68.45 & 61.41 & 59.66 & 60.95 & 65.00 & 70.84 & 93.07 & 96.98 & 97.42 & 97.66 & 98.24 & 98.48 \\
\hline
\end{tabular}


Table 2. The $L_{\text {are }}$ between the $S P_{\mathrm{N}}$ model results and MC model results.

\begin{tabular}{lllllllllllll}
\hline$L_{\text {are }}(\%)$ & 607 & 615 & 618 & 620 & 623 & 627 & 667 & 692 & 700 & 703 & 720 & 802 \\
\hline $\mathrm{DE}\left(S P_{1}\right)$ & 2.54 & 1.37 & 1.17 & 1.15 & 1.09 & 1.00 & 1.95 & 2.89 & 3.59 & 3.81 & 4.96 & 8.37 \\
$S P_{3}$ & 1.58 & 0.56 & 0.39 & 0.36 & 0.32 & 0.33 & 1.30 & 2.56 & 3.41 & 3.65 & 4.88 & 8.29 \\
$S P_{5}$ & 1.52 & 0.53 & 0.37 & 0.34 & 0.31 & 0.31 & 1.26 & 2.47 & 3.39 & 3.63 & 4.87 & 8.28 \\
$S P_{7}$ & 1.48 & 0.49 & 0.36 & 0.31 & 0.31 & 0.29 & 1.23 & 2.47 & 3.37 & 3.62 & 4.86 & 8.28 \\
\hline
\end{tabular}

Table 3. The average time-consumption of the five models.

\begin{tabular}{lccccc}
\hline Model & $\mathrm{DE}\left(S P_{1}\right)$ & $S P_{3}$ & $S P_{5}$ & $S P_{7}$ & $\mathrm{MC}$ \\
\hline Average time-consumption (s) & 23.23 & 138.92 & 430.58 & 1081.53 & 2316.57 \\
\hline
\end{tabular}

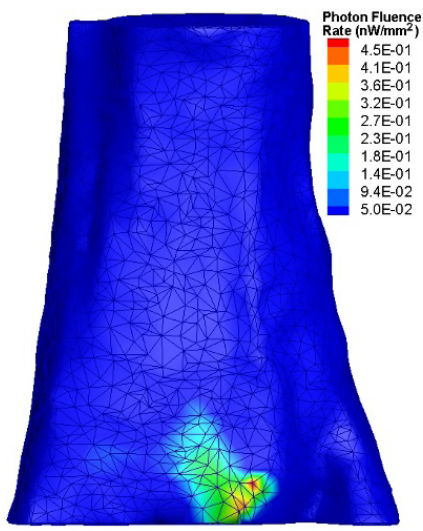

(a)

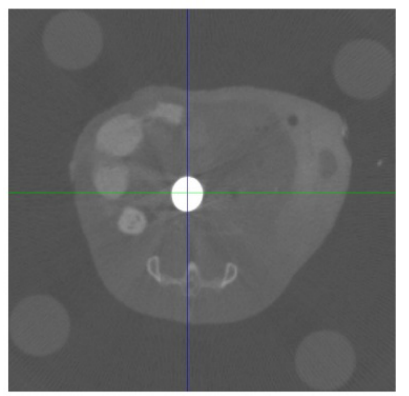

(b)
Fig. 5. (a) The distribution of forward photon on the surface. (b) The micro-CT result at $z=6.8 \mathrm{~mm}$.

Table 4. The reconstruction results of $\mathrm{DE}\left(S P_{1}\right)$ and $S P_{3}$.

\begin{tabular}{lcccc}
\hline & $\begin{array}{c}\text { Real } \\
\text { position } \\
\text { center } \\
(\mathrm{mm})\end{array}$ & $\begin{array}{c}\text { Location } \\
\text { error } \\
(\mathrm{mm})\end{array}$ & $\begin{array}{c}\text { Recovered } \\
\text { density } \\
(\mathrm{g} / \mathrm{ml})\end{array}$ & $\begin{array}{c}\text { Relative } \\
\text { quantity } \\
\text { error }(\%)\end{array}$ \\
\hline $\mathrm{DE}\left(S P_{1}\right)$ & $(12.0,13.2,6.8)$ & 1.65 & 0.009 & 40.00 \\
$S P_{3}$ & & 0.92 & 0.011 & 26.67 \\
\hline
\end{tabular}

Table 4 gives the reconstruction results of $S P_{3}$ and $\mathrm{DE}\left(S P_{1}\right)$, respectively. Figure 6 gives the transverse view of results along with corresponding CT slices $(z=6.8 \mathrm{~mm})$. As shown in Table 4 and Fig. 6, results of $S P_{3}$ have lower LE, larger recovered density and lower relative quantity error compared with $\mathrm{DE}\left(S P_{1}\right)$. The final quantitative results clearly demonstrated that $S P_{3}$ had more

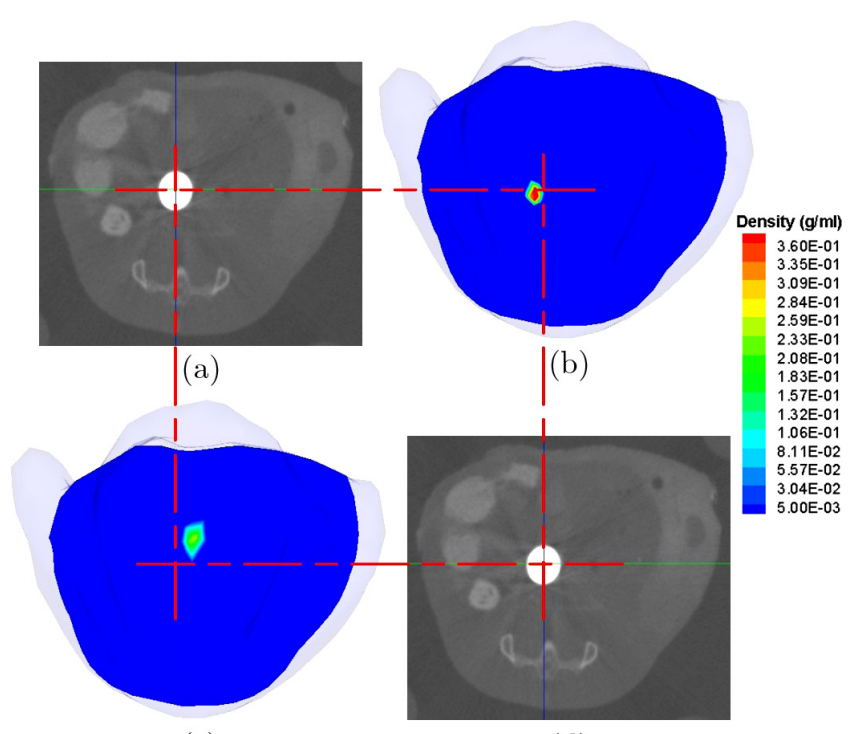

(c)

(d)

Fig. 6. The transverse view of the results along with corresponding CT slices. (b) and (c) The cross-sectional views of reconstruction results obtained by $S P_{3}$ model and $\mathrm{DE}\left(S P_{1}\right)$ model, respectively. (a) and (d) The $z=6.8 \mathrm{~mm}$ micro slices corresponding to (b) and (c).

accurate reconstruction results than $\mathrm{DE}\left(S P_{1}\right)$ had in the inverse problem of CB-XLCT.

\section{Discussion and Conclusion}

In this paper, the performance and applicability of different $S P_{\mathrm{N}}$ models of CB-XLCT were compared with MC model over all spectrums based on several main XLCT probes that have been successfully applied in this field up till now. The in vivo experiment was conducted to further demonstrate the 
reconstruction performance of the $S P_{3}$ and $\mathrm{DE}$ $\left(S P_{1}\right)$. Several useful conclusions could be drawn from the experiments. Firstly, to balance accuracy and computational efficiency, the $S P_{3}$ model was the best choice for CB-XLCT imaging based on all known probes. Secondly, $692 \mathrm{~nm}$ can be viewed as the dividing line between the $S P_{3}$ model and the DE $\left(S P_{1}\right)$ model, above which their difference was small. Finally, considering the time-consumption and ignoring their difference, the $\mathrm{DE}\left(S P_{1}\right)$ model can be employed for CB-XLCT imaging when the wavelength is above $692 \mathrm{~nm}$.

In summary, this study would provide a detailed guidance for the choice of either $S P_{3}$ or $\mathrm{DE}\left(S P_{1}\right)$ depending on different XLCT probes. Particularly, this study would also be helpful for the study of photon-transportation models for the CB-XLCT imaging. In addition, because dynamic XLCT imaging was proven useful for metabolic processes of nanophosphors-based drugs,${ }^{9}$ further research will focus on accelerating the computations of the photon-transportation model and applying them to a single-view reconstruction for fast CB-XLCT imaging.

\section{Acknowledgment}

The authors would like to thank the School of Life Science and Technology of Xidian University for providing experimental data acquisition system. This work was supported by the National Natural Science Foundation of China under Grant (Nos. 61372046, 61401264, 11571012, 61601363, 61640418, 61572400), the Science and Technology Plan Program in Shaanxi Province of China under Grant (Nos. 2013K12-20-12, 2015KW-002), the Natural Science Research Plan Program in Shaanxi Province of China under Grant (No. 2015JM6322), the Scientific Research Founded by Shaanxi Provincial Education Department under Grant No. 16JK1772, the Scientific Research Foundation of Northwest University under Grant Nos. 338050018 and 338020012.

\section{References}

1. M. Ahmad, G. Pratx, M. Bazalova, X. Lei, "X-Ray luminescence and X-ray fluorescence computed tomography: New molecular imaging modalities," IEEE Access. 2(2), 1051-1061 (2014).
2. W. Cai, X. Chen, "Nanoplatforms for targeted molecular imaging in living subjects," Small 3(11), 1840-1854 (2007).

3. G. Pratx, C. M. Carpenter, C. Sun, L. Xing, "X-ray luminescence computed tomography via selective excitation: A feasibility study," IEEE Trans. Med. Imag. 29(12), 1992-1999 (2010).

4. X. Liu, Q. Liao, H. Wang, Z. Yan, "Excitation-resolved cone-beam X-ray luminescence tomography," J. Biomed. Opt. 20(7), 70501 (2015).

5. C. Li, M. Arnuflo, S. R. Cherry, "Numerical simulation of X-ray luminescence optical tomography for small-animal imaging," J. Biomed. Opt. 19(4), 523529 (2014).

6. D. Chen, S. Zhu, X. Chen, T. Chao, X. Cao, F. Zhao, L. Huang, J. Liang, "Quantitative cone beam X-ray luminescence tomography/X-ray computed tomography imaging," Appl. Phys. Lett. 105(19), 191104 (2014).

7. L. Sudheendra, G. K. Das, C. Li, D. Stack, J. Cena, S. Cherry, I. M. Kennedy, "NaGdF4:Eu3+ nanoparticles for enhanced X-ray excited optical imaging," Chem. Mater. 26(5), 1881-1888 (2014).

8. Y. Osakada, G. Pratxd, C. Sund, M. Sakamoto, M. Ahmadd, O. Volotskovad, Q. Ongc, T. Teranishie, Y. Haradaa, L. Xing, B. Cuic, "Hard X-ray-induced optical luminescence via biomolecule-directed metal clusters," Chem. Commun. 50(27), 3549-3551 (2014).

9. X. Liu, Q. Liao, H. Wang, "Fast X-ray luminescence computed tomograph imaging," IEEE Trans. Biomed. Eng. 61(6), 1621-1627 (2014).

10. D. Wang, J. He, H. Qiao, X. Song, Y. Fan, D. Li, "High-performance fluorescence molecular tomography through shape-based reconstruction using spherical harmonics parameterization," PloS one 9(4), e94317 (2014).

11. J. Yu, J. Cheng, Y. Hou, X. He, "Sparse reconstruction for fluorescence molecular tomography via a fast iterative algorithm," J. Innov. Opt. Health Sci. 7(3), 488 (2014).

12. X. He, J. Liang, X. Wang, J. Yu, X. Qu, X. Wang, Y. Hou, D. Chen, F. Liu, J. Tian, "Sparse reconstruction for quantitative bioluminescence tomography based on the incomplete variables truncated conjugate gradient method," Opt. Express. 18(24), 24825-24841 (2010).

13. J. Feng, K. Jia, G. Yan, S. Zhu, C. Qin, Y. Lv, J. Tian, "An optimal permissible source region strategy for multispectral bioluminescence tomography," Opt. Express 16(20), 15640-15654 (2008).

14. C. Qin, J. Feng, S. Zhu, X. Ma, J. Zhong, P. Wu, Z. Jin, J. Tian, "Recent advances in bioluminescence tomography: Methodology and system as well 
as application," Laser Photon. Rev. 8(1), 94-114 (2014).

15. C. Darne, Y. Lu, E. Sevick-Muraca, "Small animal fluorescence and bioluminescence tomography: A review of approaches, algorithms and technology update," Phys. Med. Bio. 59, R1-R64 (2014).

16. S. Ren, X. Chen, H. Wang, X. Qu, G. Wang, J. Liang, J. Tian, "Molecular optical simulation environment (MOSE): A platform for the simulation of light propagation in turbid media," Plos One 8(4), e61304 (2013).

17. D. Yang, X. Chen, X. Cao, J. Wang, J. Liang, J. Tian, "Performance investigation of $S P_{3}$ and diffusion approximation for three-dimensional wholebody optical imaging of small animals," Med. Bio. Eng. Comput. 53, 805-814 (2015).

18. A. D. Klose, E. W. Larsen, "Light transport in biological tissue based on the simplified spherical harmonics equations," J. Comput. Phys. 220(1), 441-470 (2006).

19. X. He, H. Guo, J. Yu, X. Zhang, Y. Hou, "Effective and robust approach for fluorescence molecular tomography based on CoSaMP and $S P_{3}$ model," J. Innov. Opt. Health Sci. 9(6), 1650024 (2016).

20. D. Han, J. Tian, "Sparsity-promoting tomographic fluorescence imaging with simplified spherical harmonics approximation," IEEE Trans. Bio. Med. Eng. 57(10), 2564-2567 (2010).

21. A. D. Klose, B. J. Beattie, H. Dehghan, L. Vider, C. Le, V. Pronomarev, R. Blasberg, "In vivo bioluminescence tomography with a blocking-off finite-difference $\mathrm{SP}_{3}$ method and MRI/CT coregistration," Med. Phys. 37(1), 329-337 (2010).

22. D. Yang, X. Chen, Z. Peng, X. Wang, J. Ripoll, J. Wang, Liang, "Light transport in turbid media with non-scattering, low-scattering and high absorption heterogeneities based on hybrid simplified spherical harmonics with radiosity model," BioMed. Opt. Express 4(10), 2209-2223 (2013).

23. X. Chen, Q. Zhang, D. Yang, J. Liang, "Hybrid radiosity- $\mathrm{SP}_{3}$ equation based bioluminescence tomography reconstruction for turbid medium with low- and non-scattering regions," J. Appl. Phys. 115(2), 024702-1-8 (2014).

24. W. Cong, G. Wang, D. Kumar, Y. Liu, M. Jiang, L. Wang, E. Hoffman, G. Mclennan, P. Mcracy, J. Zabner, A. Cong, "Practical reconstruction method for bioluminescence tomography" Opt. Express 13(18), 6756-6771 (2005).

25. Y. Deng, Z. Luo, X. Jiang, W. Xie, Q. Luo, "Accurate quantification of fluorescent targets within turbid media based on a decoupled fluorescence Monte Carlo model," Opt. Lett. 40(13), 31293132 (2015).

26. B. Dogdas, D. Stout, A. F. Chatziioannou, R. M. Leahy, "Digimouse: A 3D whole body mouse atlas from CT and cryosection data," Phys. Med. Biol. 52(3), 577-587 (2007).

27. G. Alexandrakis, F. Rannou, A. Chatziioannou, "Tomographic bioluminescence imaging by use of a combined optical-PET (OPET) system: A computer simulation feasibility study," Phys. Med. Biol. 50(17), 4225-4241 (2005).

28. D. Hyde, R. Schulz, D. Brooks, E. Miller, V. Ntziachristos, "Performance dependence of hybrid X-ray computed tomography/fluorescence molecular tomography on the optical forward problem," J. Opt. Soc. Am. A 26(4), 919-923 (2009).

29. L. A. Feldkamp, L. C. Davis, J. W. Kress, "Practical cone-beam algorithm," J. Opt. Soc. Am. A 1(6), 612-619 (1984).

30. H. Yi, D. Chen, W. Li, S. Zhu, X. Wang, J. Liang, J. Tian, "Reconstruction algorithms based on 11norm and 12-norm for two imaging models of fluorescence molecular tomography: A comparative study," J. Biomed. Opt. 18(5), 467-472 (2013).

31. D. Chen, S. Zhu, H. Yi, X. Zhang, D. Chen, J. Liang, J. Tian, "Cone beam X-ray luminescence computed tomography: A feasibility study," Med. Phys. 40(40), 031111 (2013). 